\title{
Aseptic necrosis at multiple localisations in a lupus patient with lymphoma
}

\author{
A. Bazso • T. Bazso • P. Szodoray • G. Poor • E. Kiss
}

Received: 18 September 2013 / Accepted: 20 November 2013 / Published online: 3 December 2013

(C) The Author(s) 2013. This article is published with open access at Springerlink.com

\begin{abstract}
Avascular or aseptic necrosis is a well-defined entity leading to the degradation of cellular elements of the bone. The pathogenesis of osteonecrosis $(\mathrm{ON})$ is still unknown. There are two main types of ON: traumatic or nontraumatic. Several clinical entities could associate with ON, systemic diseases, environmental factors, pregnancy, systemic autoimmune or rheumatic diseases, thrombophilia, corticosteroid therapy, cytotoxic dugs, infections, metabolic and hematologic diseases, etc. Corticosteroids (CS) are still the most frequently used therapeutic options in the early phase and during flares of these diseases. Inflammatory cytokines and antibodies have been described to participate in the pathogenesis of ON. The infiltrative disorders of the bone marrow could also contribute to the development of ON. Hereby, we describe a female patient with NHL followed by SLE in whom ON has developed at least in two localisations. Lupus flare, long-term CS therapy, lymphoma relapse or the presence of antiphospholipid antibodies were excluded. Although the bi-localised ON could be contributed to immunologic factors or trauma, the exact aetiology in this case could not be elucidated.
\end{abstract}

A. Bazso $\cdot$ G. Poor $\cdot$ E. Kiss

National Institute of Rheumatology and Physiotherapy, Budapest, Hungary

T. Bazso

Department of Orthopaedic Surgery, University of Debrecen, Medical and Health Science Center, Medical School, Debrecen, Hungary

P. Szodoray

Institute of Immunology, Rikshospitalet, University of Oslo, Oslo, Norway

A. Bazso $(\bowtie)$

Frankel Leo u. 38-40, 1023 Budapest, Hungary

e-mail: bazsoanna@yahoo.com
Keywords Antiphospholipid antibodies · Aseptic necrosis · Corticosteroid $\cdot$ NHL $\cdot$ SLE

Avascular or aseptic necrosis is a well-defined entity leading to the degradation of cellular elements of the bone. The pathogenesis of osteonecrosis $(\mathrm{ON})$ is still unknown. There are two main types of ON: traumatic or non-traumatic. Several clinical entities could associate with ON, systemic diseases, environmental factors, pregnancy, systemic autoimmune or rheumatic diseases, thrombophilia, corticosteroid therapy, cytotoxic dugs, infections, metabolic and hematologic diseases, etc. There are some systemic autoimmune diseases, such as systemic lupus erythaematosus (SLE), antiphospholipid syndrome (APS) and vasculitis which may associate more frequently with ON than others (1). Corticosteroids (CS) are still the most frequently used therapeutic options in the early phase and during flares of these diseases (2). Inflammatory cytokines and antibodies have been described to participate in the pathogenesis of $\mathrm{ON}$. The infiltrative disorders of the bone marrow could also contribute to the development of $\mathrm{ON}$; however, in non-Hodgkin's lymphomas (NHL), no clear association with ON have been shown previously (1). Hereby, we describe a female patient with NHL followed by SLE in whom ON has developed at least in two localisations. Lupus flare, long-term CS therapy, lymphoma relapse or the presence of antiphospholipid antibodies were excluded. Although the bi-localised ON could be contributed to immunologic factors or trauma, the exact aetiology in this case could not be elucidated.

We report a case of a 32-year-old Caucasian woman with large B cell mediastinal NHL treated with radiotherapy and autologous bone marrow transplantation. Following the therapy, she got in remission. Later, at the age of 40, she complained for fever and polyarthritis. The relapse of the NHL was suspected therefore positron emission tomography 
(PET) was performed with normal result. Besides polyarthritis, ANA, a-dsDNA, leukopenia and anaemia developed; SLE was diagnosed. She was treated with short-term high-dose CS, followed by low-dose CS, only for a 1-year period (preventing flares), and chloroquine as maintenance therapy was administered. Brain magnetic resonance imaging (MRI), which was performed due to migraine, detected few small, micro-lacunar, ischemic, micro-vascular lesions, which could be considered as vasculitis. In January 2012, she complained for severe right hip pain. Aseptic necrosis of the femur head was verified with MRI. Because of the movement restriction and persistent pain, a total endoprothesis was implanted into the right hip. Histopathology was consistent with aseptic osteonecrosis without any signs of malignancy either in the bone cortex or bone marrow spaces. The surgical intervention was completed with a complex rehabilitation. In June 2011, severe left shoulder pain developed. The MRI showed several subchondral lythic and sclerotic spaces with particular fractures in the head of the humerus, characteristic findings for aseptic bone necrosis. Both native CT, FDG-PET and laboratory investigations excluded malignancy, infection or the reoccurrence of NHL. There were no clinical or laboratory signs indicating relapse of SLE. None of the antiphospholipid antibodies were detected since disease onset. Quantiferon tests for tuberculosis were negative. The serum bone turnover biochemical markers and parathormone, TSH, and also 25-OH vitamin D level were normal. Dual X-ray absorptiometry showed mild osteopenia. The patient did not drink alcohol habitually; there was no evidence for hyperlipidaemia or coagulopathy.

Since the patient received high-dose CS only in the early phase of the disease (SLE) for remission inductions and CS was gradually tapered then omitted, the treatment could not be the cause for the aseptic ON. Presently, she is a recipient for prosthesis of the left shoulder and also for arthroscopy or synovectomy of the left elbow.

$\mathrm{ON}$ is a clinical feature leading to the degradation of bone marrow and trabecular bone, resulting in the collapse of bone architecture. Clinically, it is manifested in bone and joint pain, bone destruction and loss of function, although it could remain silent for a long time. However, the pain also can develop rapidly and can be very intensive, especially when caused by large infarcts of the femoral head. The aseptic, or as a synonym, avascular necrosis may be caused by several factors, including local trauma or non-traumatic systemic diseases. The predisposing factors are alcoholism; iatrogenic agents; pancreatitis; hematologic, metabolic or endocrine disorders; pregnancy; neoplastic diseases; systemic autoimmune; rheumatic diseases; or orthopaedic disorders. Approximately, in $75 \%$ of the cases, ON starts between the ages of 30 and 60 years. The frequency of avascular necrosis in SLE ranges from 3 to $30 \%$; however, a higher frequency (40\%) was reported in children and young patients with lupus (1).
$\mathrm{CS}$ are reported as major risk factors for development of ON in SLE, where steroids are usually the most frequently applied treatment options in the early phase or flares of the disease. ON occurs mainly in SLE patients who are treated with CS and is extremely rare among those who have never received CS $(2,3)$. However, another retrospective study has shown no relationship between the risk of ON development and the total dose, duration of steroid therapy and disease activity (4). However, vasospasm, cushingoid feature, vasculitis, smoking, pre-eclampsia and the presence of lupus anticoagulant are suspected to be associated with increased risk of ON (4).

SLE is often associated with APS (5). In our case, the patient had no presence of antiphospholipid antibodies [anticardiolipin antibody ( $\mathrm{aCL}$ ), lupus anticoagulant and anti- $\beta 2$ glycoprotein-I]. There are some case reports and studies describing the strong association between primary and secondary APS and ON $(6,7)$. However, no difference in the prevalence of aCL in patients with or without $\mathrm{ON}$ were found previously $(8,9)$. A clearly increased risk for NHL has been described in patients with SLE as compared to those in the general population. It has still remained unclear to what extent the association with NHL is mediated by innate versus exogenous factors $(10,11)$. Interestingly, diffuse, large B cell lymphoma was the first disease in our case preceding lupus. Therefore, paraneoplastic syndrome had to be accounted, although lymphoma was confirmed being in remission when SLE developed. Primary or metastatic bone cancer was excluded in our case and bone turnover biomarkers were normal. In myeloma multiplex (MM), osteoclast activation and osteoblast inhibition have been described (12). Myeloma cells lead to an imbalance in the receptor activator of nuclear factorkappa B/receptor activator of nuclear factor-kappa B ligand (RANK/RANK-L)-system in the tumour microenvironment, such as the receptor activator of nuclear factor-kappa B ligand/ osteoprotegerin (RANK-L/OPG) ratio is essential for normal bone turnover. Myeloma cells induce RANK-L expression and decrease OPG secretion by osteoblasts and stromal cells $(13,14)$. There are other pathways of MM in the development of $\mathrm{ON}$, as myeloma cells produce and shed syndecan (CD 138) that binds to the heparin-binding domain of OPG and mediates its internalisation and lysosomal degradation by myeloma cells. Other cytokines, as MIP- $1 \alpha$ and MIP- $1 \beta$ enhance RANK-L expression in stromal cells. MIP- $1 \alpha$ promotes growth, survival and migration of myeloma cells (15). There are two basic and common cytokines, interleukin (IL)-6 and IL-11, which are predominantly produced by stromal cells, as well as inflammatory cells involved in SLE (16). Both the upregulation of IL-6 secretion through cell-to-cell contact and the downregulation of OPG result in increased osteoclast activation and a consequent bone resorption. Less defined in lupus, but the IL-3, hepatocyte growth factor (HGF), SDF- $1 \alpha$ and its receptor CXCR4 are expressed on 
osteoclast precursors, which all increase osteoclast motility and bone-resorpting activity. Other similar ON-provoking factors, as MMP-1 and MMP-2, urokinase plasminogen activator and HGF, are recognised both in MM and lupus $(17,18)$. Presumably, these factors may lead to avascular necrosis of the bone in NHL. Bernbeck et al. described bone marrow oedema and aseptic necrosis in children and adolescent with acute lymphoblastic leukaemia or NHL treated with hyperbaric oxygen therapy, whereas the $\mathrm{ON}$ was contributed to the treatment rather than the haematologic disease (19). In MM, there are some promising therapeutic options, as antibodies to MIP- $1 \alpha$ and MIP- $1 \beta$ or DKK- 1 gene produced protein which could be also effective in lupus-associated ON (20). Anti-IL-6 and anti-IL-3 may be common therapeutic options in lupus and associated ON $(16,21)$, while shared therapeutic targets between lupus and myeloma, such as proteasome inhibitors also can be considered in these patients $(22,23)$. We believe that the development of $\mathrm{ON}$ in our patient was due to a systemic immune activation, pro-inflammatory cytokine secretion and presumably altered RANK/RANKL system. Targeting pro-inflammatory cytokines and anti-resorptive therapy can be beneficial in lupus patients with $\mathrm{ON}$.

\section{Conflicts of interest None.}

Open Access This article is distributed under the terms of the Creative Commons Attribution Noncommercial License which permits any noncommercial use, distribution, and reproduction in any medium, provided the original author(s) and the source are credited.

\section{References}

1. Powell C, Chang C, Naguwa SM et al (2010) Steroid induced osteonecrosis: an analysis of steroid dosing risk. Autoimmune Rev 9:721-743

2. Seguro LP, Rosario C, Shoenfeld Y (2013) Long-term complications of past glucocorticoid use. Autoimmune Rev 12:629-632

3. Zonana Nacach A, Barr SG, Magder LS et al (2000) Damage in systemic lupus erythematosus and its association with corticosteroids. Arthritis Rheum 43:1801-1808

4. Assouline-Dayan Y, Chang C, Greenspa A et al (2002) Pathogenesis and natural history of osteonecrosis. Semin Arthritis Rheum 32:94124

5. Love PE, Santoro SA (1990) Antiphospholipid antibodies: anticardiolipin and the lupus anticoagulant in systemic lupus erythaematosus (SLE) and in non-SLE disorders; prevalence and clinical significance. Ann Intern Med 112:682-698

6. Rueda JC, Duque MA, Mantilla RD et al (2009) Osteonecrosis and antiphospholipid syndrome. J Clin Rheumatol 15:130-132
7. Zonana-Nacach A, Jiménez-Balderas FJ (2004) Avascular necrosis of bone associated with primary antiphospholipid syndrome: case report and literature review. J Clin Rheumatol 10:214-217

8. Houssiau FA, N'Zeusseu Toukap A, Depresseux G et al (1998) Magnetic resonance imaging-detected avascular osteonecrosis in systemic lupus erythematosus: lack of correlation with antiphospholipid antibodies. Br J Rheumatol 37:448-453

9. Migliaresi S, Picillo U, Ambrosone L et al (1994) Avascular osteonecrosis in patients with SLE: relation to corticosteroid therapy and anticardiolipin antibodies. Lupus 3:37-41

10. Tarr T, Gyorfy B, Szekanecz E, Bhattoa HP, Zeher M, Szegedi G, Kiss E (2007) Occurrence of malignancies in Hungarian patients with systemic lupus erythematosus. Results from a single centre. Ann NY Acad Sci 1108:76-82

11. Kiss E, Kovacs L, Szodoray P (2010) Malignancies in systemic lupus erythematosus. Autoimmun Rev 9(4):195-199

12. Sezer O (2009) Myeloma bone disease: recent advances in biology, diagnosis, and treatment. The Oncoogist 14:276-283

13. Giuliani N, Bataille R, Mancini C et al (2003) Myeloma cells induce imbalance in the osteoprotegerin/osteoprotegerin ligand system in the human bone marrow environment. Blood 98: $3527-3533$

14. Heider U, Langelotz C, Jakob C et al (2003) Expression of receptor activator of nuclear factor kappaB ligand on bone marrow plasma cells correlates with osteolytic bone disease in patients with multiple myeloma. Clin Cancer Res 9:1436-1440

15. Abe M, Hiura K, Wilde J et al (2002) Role of macrophage inflammatory protein (MIP)- $1 \alpha$ and MIP- $1 \beta$ in the development of osteolytic lesions in multiple myeloma. Blood 100: 2195-2202

16. Sun LY, Zhang HY, Feng XB et al (2007) Abnormality of bone marrow-derived mesenchymal stem cells in patients with systemic lupus erythematosus. Lupus 16:121-128

17. Zannettino AC, Farrigia AN, Kortesidis A et al (2005) Elevated serum levels of stromal-derived factor- $1 \alpha$ are associated with increased osteoclast activity and osteolytic bone disease in multiple myeloma patients. Cancer Res 65:1700-1709

18. Toldi G, Szalay B, Bekő G, Bocskai M et al (2012) Plasma soluble urokinase plasminogen activator receptor (suPAR) levels in systemic lupus erythematosus. Biomarkers 17:758-763

19. Bernbeck B, Christaras A, Krauth K et al (2004) Bone marrow oedema and aseptic osteonecrosis in children and adolescents with acute lymphoblastic leukaemia or non-Hodgkin-lymphoma treated with hyperbaric-oxygen-therapy (HBO): an approach to cure?$\mathrm{BME} / \mathrm{AON}$ and hyperbaric oxygen therapy as a treatment modality. Klin Padiatr 216:370-378

20. Heider U, Kaiser M, Mieth M et al (2009) Serum concentrations of DKK-1 decrease in patients with multiple myeloma responding to anti-myeloma treatment. Eur J Haematol 82:31-38

21. Alcocer-Varela, Vidaller A, Llorente L (1988) Presence of an IL-3producing suppressor $\mathrm{T}$ cell resistant to cyclosporin $\mathrm{A}$ in the peripheral blood of patients with systemic lupus erythematosus. Clin Exp Immunol 73:424-429

22. Paz Z, Tsokos GC (2013) New therapeutics in systemic lupus erythematosus. Curr Opin Rheumatol 25:297-303

23. Lee HC, Shah JJ, Orlowski RZ (2013) Novel approaches to treatment of double-refractory multiple myeloma. Am Soc Clin Oncol Educ Book 2013:302-306 